\title{
Editorials
}

\section{First, do no harm: balancing the risks and benefits of regional anesthesia in patients with underlying neurological disease}

Joanne Guay MD FRCPC

$\mathrm{P}$ ERMANENT neurological injury is a complication of regional anesthesia feared by all anesthesiologists. Fortunately, such permanent injury has become quite rare. If hematoma and abscess are excluded, the absolute incidence, in adults, of permanent neurological injury per 10,000 procedures, is between 0 and 4.2 events for spinal anesthesia and between 0 and 7.6 events for epidural anesthesia. ${ }^{1}$ For peripheral nerve blocks, a current estimate of the incidence of permanent damage is lacking. However, when reviewing the closed anesthesia malpractice insurance claim files of more than 35 professional liability companies in the United States (for the years 1980 to 1999), Lee et al. ${ }^{2}$ found only 39 cases of permanent nerve injury related to peripheral nerve blocks, when eye blocks were excluded. When a nerve injury is observed after a surgical procedure, the anesthetic technique is responsible in a minority of cases. When reviewing a total of 1,614 axillary blocks performed in 607 patients, Horlocker et al. ${ }^{3}$ found that only $11.3 \%$ of the nerve injuries $(7 / 62)$ could be related to the anesthetic technique. Surgical variables were considered to have been responsible in $88.7 \%$ of the neurological complications identified, namely: direct surgical trauma or stretch, inflammation or infection, hematoma or vascular compromise, cast irritation, and tourniquet ischemia. Furthermore, in this retrospective study, all complications involving motor deficits had a surgical cause.

Mechanisms involved in regional anesthesia-related neurological injury include: laceration (direct needle trauma), pressure disruption or compression, ischemia, drug neurotoxicity, and secondary inflamma- tion and scarring. While a short-bevel needle $\left(27^{\circ}\right)$ is less likely to enter the nerve than a long-bevel needle $\left(12^{\circ}\right)$, it generally induces more damage when it does so. ${ }^{4}$ Experimentally, intraneural (within the perineurium) local anesthetic injection would be associated with long term (> $24 \mathrm{hr}$ ) damage, only if associated with a high pressure injection (cutoff point around 25 psi). ${ }^{4}$ A cytotoxic effect of local anesthetics can be demonstrated at clinical concentrations. By stopping mitochondrial adenosine triphosphate production, local anesthetics provoke failure of axonal transportation of the material synthetized by the perikaryon (reduced axoplasmic flow), as well as intracellular calcium accumulation, with subsequent enzyme activation and cell degradation. ${ }^{4}$ Local anesthetics may also act as a "detergent" and cause nerve membrane solubilization. ${ }^{4}$

Historically, regional anesthesia has always been considered relatively contraindicated in patients with an underlying neurological disease. Sustaining these concerns is the likelihood of the "double-crush phenomenon" or the theoretically increased susceptibility of a partially unsheathed nerve (myelin damage) to local anesthetics being invoked. But how do these phenomena really apply to regional anesthesia? The "double-crush phenomenon" refers to the fact that partial reductions of the axonomic flow, at two different levels (nerve entrapment at two different levels), would have an additive, or even a synergistic, effect. ${ }^{5}$ At least two studies have evaluated the effect of peripheral nerve blocks on patients with mechanical nerve lesions. Hebl et al. ${ }^{6}$ compared two groups of patients who had ulnar nerve transposition under

CAN J ANESTH 2008/55: 8 / pp 489-494

From the Department of Anesthesia, Maisonneuve-Rosemont Hospital, University of Montreal, Montreal, Quebec, Canada. Address correspondence to: Dr. Joanne Guay, Clinical Associate Professor, Department of Anesthesia, Maisonneuve-Rosemont Hospital, 5415, L'Assomption Boulevard, Montreal, Quebec H1T 2M4, Canada. Phone: 514-252-3426, Fax: 514-252-3542, E-mail: joanne.guay@umontreal.ca Competing interests: None. 
regional $(n=100)$ or general anesthesia $(n=260)$. The anesthetic technique did not influence the incidence of new or worsening, neurological symptoms, either immediately after the surgery, or at two or six weeks, postoperatively. ${ }^{6}$ Likewise, in 273 consecutive patients, wrist blocks for carpal tunnel release were not associated with any anesthesia-related neurological symptoms, at $24 \mathrm{hr}$ or at one week. ${ }^{7}$ With these two studies, one can probably conclude that surgeries for nerve entrapment or nerve transposition are not valid contraindications to regional anesthesia.

The situation might not be as clear for neurological injuries from other causes. For example, in a 14-yr-old girl who had previously received a high cumulative dose $\left(840 \mathrm{mg} \cdot \mathrm{m}^{-2}\right)$ of cisplatin chemotherapy, a case of severe brachial plexopathy has been reported 48 hr after an interscalene block performed with $0.5 \%$ bupivacaine with epinephrine. The addition of two different, potentially neurotoxic drugs might have been responsible for the neurological injury in this patient. $^{8}$

In this issue of the Journal, Dhir et al. ${ }^{9}$ report successful outcomes with four ultrasound-guided axillary, brachial plexus blocks, in three patients with Charcot-Marie-Tooth disease. Ultrasound guidance was thought to have facilitated needle localization, and none of the three patients experienced any worsening of his neurological condition. Very recently, Koff et al. ${ }^{10}$ reported a case of severe brachial plexopathy occurring in a patient with multiple sclerosis, after an ultrasound-guided interscalene block; they suggested that the underlying neurological disorder might have contributed to the injury. Who's right, and who's wrong? Is it reasonable to assume a quantifiably small, but unknown, added risk of performing regional anesthesia in patients with underlying inflammatory or congenital neurological disease? Will ultrasoundguided techniques confer any higher degree of patient safety with these co-morbidities?

As mentioned by Dhir et al., ${ }^{9}$ ultrasound guidance may facilitate nerve localization in patients who respond poorly to electrostimulation (decrease motor contraction amplitude to a predetermined stimulus), and one may be more tempted to comply with a patient's strong preference towards regional anesthesia if ultrasound guidance is available. There are no data, however, which unequivocally demonstrate that ultrasound guidance will make regional anesthesia safer in these patients. Visualization of the needle tip is frequently lost while the block is being performed, and this may occur even in the hands of experienced (more than 100 blocks performed) operators. ${ }^{11}$ In certain anatomic locations, nerves cannot be visual- ized with this technology. ${ }^{12}$ As a result, inadvertent neural puncture and intraneural injections are not uncommon occurrences when peripheral nerve blocks are performed under real-time ultrasound guidance. ${ }^{13}$ Finally, if the neurological injury occurs because the nerve is more susceptible to the toxicity of the local anesthetic (concentration and exposure-duration dependent), the risk might well remain the same, no matter how the block is performed.

However, should we refrain from performing regional anesthesia in all patients with underlying neurological disease? This belief may be challenged by three retrospective studies. The first of these studies examined neuraxial blocks in patients with central nervous system disease and includes 139 patients, 79 of whom had a post-poliomyelitis syndrome, and 35 of whom had multiple sclerosis. ${ }^{14}$ Preoperatively, $53 \%$ of the patients experienced active neurologic symptoms, including motor or sensory deficits, dysesthesias/paresthesias, or hyperreflexia. In $96 \%$ of these patients, the underlying neurological conditions had been stable for at least six months. Eighty-one patients received a spinal anesthetic, and 58 received an epidural, either for operative anesthesia $(n=39)$ or for labour or postoperative analgesia. Bupivacaine $0.75 \%$ was the most commonly used drug for the spinals, at a mean dose of $14 \pm 2 \mathrm{mg}$ (range 7.5-22.5 $\mathrm{mg}$ ). A worsening of the neurological condition was not observed in any patient. From this study, the incidence of neurological complication, after a neuraxial block in patients with underlying central nervous system pathology, was estimated to be between 0 and 30 per 10,000 procedures. A second study examined outcomes with neuraxial blocks in patients with peripheral neurological disease. ${ }^{15}$ The study included 567 patients with either a sensorimotor, peripheral neuropathy $(n=293)$ or a diabetic polyneuropathy $(n=274)$. Two patients experienced severe postoperative neurological complications (one with spinal anesthesia and one with an epidural used intra- and postoperatively). The two patients belonged to the diabetic polyneuropathy group. New or progressive postoperative neurological deficits, when compared with the preoperative findings, were not observed in any patient with other types of sensorimotor peripheral neuropathy. ${ }^{15}$ From this study, the incidence of neurological complications associated with a neuraxial block, in patients with underlying peripheral neurological diseases, was estimated to be between 10 and 130 per 10,000 procedures (higher than for the general population). Finally, in a study reporting 607 patients who underwent 1,614 axillary blocks, the presence of a preexisting neurological condition $(247 / 607)$ 
was not found to be a risk marker for developing an anesthesia-related neurological complication. More specifically, an underlying sensorimotor peripheral neuropathy was present in 18 of the 607 patients, and none of these patients developed an anesthesia-related neurological complication. ${ }^{3}$ Enthusiasm related to these favourable outcomes must, however, be tempered by the adverse outcome reported by Koff et al. ${ }^{10}$ In this particular case, a patient with underlying multiple sclerosis experienced a severe brachial plexopathy after an interscalene block. Adverse outcomes were also reported in two patients by Aldrete et al. ${ }^{16}$ One of these two patients with multiple sclerosis was almost asymptomatic prior to the surgery. He had received four previous spinal anesthetics with bupivacaine 10 $\mathrm{mg}$ it without any problems, but he remains unable to ambulate three years after a spinal anesthetic with a $15 \mathrm{mg}$ dose of bupivacaine. Another patient, who had mild bilateral lower limb motor weakness secondary to poliomyelitis, suffered a permanent loss of motor function, up to $\mathrm{L}_{3}$ on the left side and up to $\mathrm{L}_{1}$ on the right side, after an epidural was performed for an open reduction of a left femoral shaft fracture.

Finally, before more widespread use of continuous peripheral nerve blocks should be contemplated in patients with underlying neurological or muscular disease, the myotoxicity of local anesthetics will need further clarification. ${ }^{17}$ Local anesthetics have the potential to induce myonecrosis. This problem is rarely clinically relevant in normal patients, except at the level of the extraocular muscles (of relevance for peri- and retrobulbar blocks). Since, in most cases, myoblasts and connective tissue elements remain intact, the phenomenon is usually almost completely reversible, with muscle regeneration occurring within three to four weeks. However, the effects of local anesthetic infusions on abnormal muscles are unknown.

In conclusion, it seems possible that patients with underlying neurological disease, including those with severe diabetic polyneuropathy, are at increased risk of neurological complications from regional anesthesia. The risks may vary according to the type and severity of disease. However, it is not possible to quantify the magnitude of the increased incidence, as powering definitive, randomized controlled trials to the primary outcome of interest would probably require thousands of subjects, and additional ethical complexities related to randomization would make such studies unfeasible. Retrospective studies have inherent methodological limitations, but still provide the best available evidence. At the same time, case reports/case series, such as reported in this issue of the Journal, provide feasibility assessments from single experiences. ${ }^{9}$ When con- sidering the best available evidence related to choice of anesthetic technique in patients with neurological disease, one must also consider that general anesthesia is not devoid of potential complications in these patients. Patients with Charcot-Marie-Tooth disease have been reported to have had uneventful outcomes under regional anesthesia (with both peripheral nerve and neuraxial blocks) or under general anesthesia. ${ }^{9,18}$ However, in a patient with Charcot-Marie-Tooth disease, mechanical ventilation was required for nearly a month following a simple Cesarean delivery under general anesthesia. ${ }^{19}$ Finally, operator training and experience must be considered in deciding whether or not ultrasound guidance of regional anesthetic techniques will confer a benefit in patients with an underlying neurological disease.

\section{Prudence est mère de sureté: évaluer le ratio bénéfice/risque de l'anesthésie régionale chez les patients porteurs de pathologie neurologique}

L'atteinte neurologique permanente est une complication redoutée par tout anesthésiologiste pratiquant l'anesthésie régionale. Fort heureusement, elle semble actuellement devenue rare. Chez l'adulte, si on exclut les hématomes et les abcès, l'incidence de séquelles neurologiques permanentes par 10000 procédures varie entre 0 et 4,2 pour la rachianesthésie et 0 et 7,6 pour la péridurale. ${ }^{1}$ On ne dispose pas d'estimé à jour pour les blocs de nerfs périphériques. Toutefois, lorsqu'ils ont révisé les cas de litiges médico-juridiques pour lesquels une entente est intervenue de 35 compagnies d'assurance américaines pour les années 1980-1999, en excluant les blocs oculaires, Lee et coll. ${ }^{2}$ n'ont retrouvé que 39 cas de dommages neurologiques permanents reliés aux blocs nerveux périphériques. Lorsqu'une lésion neurologique survient suite à un acte chirurgical, l'anesthésie n'est impliquée que 
dans une minorité des cas. Dans leur revue de 1614 blocs axillaires faits chez 607 patients, Horlocker et coll. ${ }^{3}$ diagnostiquent une étiologie anesthésique pour seulement $11,3 \%(7 / 62)$ des lésions neurologiques observées. Des facteurs chirurgicaux sont à l'origine de $88,7 \%$ des cas nommément: le traumatisme chirurgical direct, l'étirement, l'inflammation ou l'infection, l'hématome ou l'ischémie vasculaire, le plâtre et le garrot. De plus, tous les cas avec atteinte de la fonction motrice avaient une origine chirurgicale.

Parmi les mécanismes impliqués dans la lésion neurologique reliée à l'anesthésie régionale on retient: la lacération par traumatisme direct de l'aiguille, le barotraumatisme entraînant soit un éclatement tissulaire ou une compression, l'ischémie, la neurotoxicité de la substance injectée ainsi que l'inflammation et la cicatrisation secondaires. Une aiguille à biseau court $\left(27^{\circ}\right)$ pénètre moins souvent le tissu nerveux qu'une aiguille à biseau long $\left(12^{\circ}\right)$, mais induit un dommage plus substantiel lorsqu'elle y parvient. ${ }^{4}$ Expérimentalement, l'injection intraneurale (à l'intérieur du périnèvre) d'un anesthésique local induit une lésion persistante (> $24 \mathrm{~h}$ ) seulement si elle s'accompagne d'une pression d'injection élevée (seuil autour de 25 psi). ${ }^{4}$ Les anesthésiques locaux sont cytotoxiques à concentration clinique. En stoppant la production mitochondriale d'adénosine triphosphate, ils provoquent un arrêt du transport axonal du matériel synthétisé par le neurone (réduction du flot axonal) ainsi qu'une accumulation intracellulaire de calcium donnant lieu à une activation enzymatique avec dégradation cellulaire subséquente. ${ }^{4}$ Ils peuvent aussi agir comme "détergent » et solubiliser la membrane nerveuse. ${ }^{4}$

Historiquement, l'anesthésie régionale a souvent été considérée relativement contre-indiquée chez les patients porteurs de pathologie neurologique. Le phénomène de la «double-insulte» et une susceptibilité accrue à la toxicité de l'anesthésique local d'un nerf partiellement démyélinisé peuvent être invoqués pour justifier cette crainte. Mais à quel point ces phénomènes s'appliquent-ils à l'anesthésie régionale? Initialement, le phénomène de la "double-insulte » a été décrit pour expliquer que des compressions nerveuses à plus d'un niveau auraient un effet additif voir même synergique sur la réduction du flot axonal. ${ }^{5}$ Deux études ont évalué l'effet de l'anesthésie régionale sur des nerfs avec lésions mécaniques. Hebl et coll. ${ }^{6}$ ont comparé deux groupes de patients subissant une transposition du nerf cubital sous anesthésie régionale $(n=$ $100)$ ou générale $(n=260)$. L'incidence de nouvelles lésions neurologiques ou d'aggravation était la même pour les deux techniques anesthésiques aussi bien immédiatement après la chirurgie qu'à deux et à six semaines. De même, lorsque des blocs du poignet ont été faits pour des décompressions du tunnel carpien chez 273 patients consécutifs, on n'a pas retrouvé de lésions neurologiques attribuables à la technique anesthésique à $24 \mathrm{~h}$ ou à une semaine après la chirurgie. ${ }^{7}$ Avec ces deux études, on peut probablement conclure que la transposition ou la décompression nerveuse ne constituent pas des contre-indications à l'anesthésie régionale.

La situation n'est peut-être pas aussi claire pour les autres types de neuropathie. Par exemple, une plexopathie brachiale survenant $48 \mathrm{~h}$ après un bloc interscalénique fait avec de la bupivacaïne 0,5 \% adrénalinée est rapportée chez une adolescente de 14 ans ayant préalablement reçu une forte dose cumulative de cisplatine $\left(840 \mathrm{mg} \cdot \mathrm{m}^{-2}\right)$. Les effets additifs de deux neurotoxiques différents pourraient être responsables de l'atteinte neurologique de cette patiente. ${ }^{8}$

Dans ce numéro du Journal, Dhir et coll. ${ }^{9}$ rapportent quatre blocs du plexus axillaire échoguidés réalisés avec succès chez trois patients porteurs de la maladie de Charcot-Marie-Tooth. Les auteurs estiment que l'utilisation des ultrasons a facilité la localisation nerveuse et aucun patient n'a subi d'aggravation de sa condition neurologique. Récemment, Koff et coll. ${ }^{10}$ rapportaient une plexopathie brachiale sévère suite à un bloc interscalénique échoguidé réalisé chez un patient porteur de sclérose en plaques et suggéraient que la condition sous-jacente ait pu contribuer à l'atteinte. Qui a raison, qui a tort? Est-il raisonnable d'assumer qu'il existe une augmentation faible mais de quantité inconnue du risque de complications neurologiques associées à l'anesthésie régionale chez les patients porteurs de pathologie neurologique inflammatoire ou congénitale et le guidage par ultrasons apportera-t-il un élément de sécurité supplémentaire pour ces patients?

Comme l'ont souligné Dhir et coll..$^{9}$ le guidage par ultrasons peut faciliter la localisation d'un nerf susceptible de moins bien répondre à la stimulation électrique (diminution de l'amplitude de la contraction musculaire à un stimulus prédéterminé) et on pourrait être plus tenté de se soumettre au désir d'un patient qui insiste pour être opéré sous anesthésie régionale si le guidage par ultrasons est disponible. Il n'y a actuellement pas de données démontrant que les ultrasons diminueront les risques de l'anesthésie régionale dans cette population. La visualisation du bout de l'aiguille est fréquemment perdue durant la procédure et ceci peut arriver même à des opérateurs expérimentés (plus de 100 blocs réalisés). ${ }^{11}$ De plus les nerfs ne sont pas visibles pour tous les sites anatomiques avec cette technologie. ${ }^{12}$ En conséquence, la ponction et l'injection 
intraneurales accidentelles ne sont pas des événements rares lors de la réalisation des blocs échoguidés. ${ }^{13}$ Finalement, si la lésion neurologique est due à la toxicité de l'anesthésique local (reliée à la concentration et à la durée d'exposition) le risque pourrait bien être le même quel que soit la technique utilisée pour réaliser le bloc.

Devrions-nous alors nous abstenir de toute technique d'anesthésie régionale chez tout patient porteur de maladie neurologique? Cette croyance peut être remise en question par trois études rétrospectives. Dans la première, 139 patients porteurs d'une pathologie du système nerveux central dont 75 d'un syndrome post-poliomyélitique et 35 d'une sclérose en plaques ont eu des blocs périmédullaires. ${ }^{14}$ Avant la chirurgie, $53 \%$ des patients rapportaient des symptômes neurologiques actifs incluant des déficits moteurs ou sensitifs, des dysesthésies/paresthésies ou une hyperréflexie. La condition neurologique était stable depuis six mois ou plus pour $96 \%$ des patients. Quatre-vingt un patients ont eu une rachianesthésie et 58 une péridurale soit pour la chirurgie $(n=39)$ ou pour le soulagement de la douleur du travail ou de la douleur postopératoire. La bupivacaïne 0,75\% était l'agent le plus souvent utilisé à une dose moyenne de $14 \pm 2 \mathrm{mg}$ (de 7,5 à 22,5 mg). Aucun patient n'a eu de détérioration neurologique. Dans cette étude, l'incidence de complication neurologique reliée à une anesthésie périmédullaire chez un patient porteur de pathologie du système nerveux central est estimée entre 0 et 30 par 10000 procédures. La seconde étude concerne aussi les blocs périmédullaires mais cette fois chez des patients porteurs de pathologie du système nerveux périphérique et inclut 567 patients avec pathologie périphérique sensitivomotrice $(n=293)$ ou une polyneuropathie diabétique $(n=274)$. Une complication neurologique sévère est retrouvée chez deux patients (une après rachianesthésie et une après péridurale utilisée en per- et en postopératoire). Les deux patients font partie du groupe polyneuropathie diabétique. Aucun patient du groupe pathologie périphérique sensitivomotrice n'a de nouvelle atteinte ou de progression de sa condition neurologique. ${ }^{15}$ De cette étude, l'incidence de complication neurologique après anesthésie périmédullaire chez un patient porteur d'une pathologie du système nerveux périphérique est estimée entre 10 et 130 par 10000 procédures, donc plus élevée que celle de la population générale. Enfin une étude effectuée chez 607 patients ayant reçu 1614 blocs axillaires n'identifie pas une condition neurologique sous-jacente $(247 / 607)$ comme marqueur de risque pour développer une complication neurologique reliée à l'anesthésie. Plus spécifiquement, une pathologie neurologique sensitivomotrice était présente chez 18 de ces 607 patients et aucun d'eux n'a développé de complication neurologique reliée à l'anesthésie. ${ }^{3}$ L'enthousiasme que pourrait susciter ces bons résultats doit toutefois être tempéré à la fois par le patient de Koff et coll..$^{10}$ (sclérose en plaques sous-jacente) chez qui une plexopathie brachiale sévère survient après un bloc interscalénique et par deux patients rapportés par Aldrete et coll. ${ }^{16}$ Le premier, porteur d'une sclérose en plaques mais quasi asymptomatique avant la chirurgie est toujours non-ambulant trois ans après une rachianesthésie avec $15 \mathrm{mg}$ de bupivacaïne. Le patient avait pourtant précédemment eu trois rachianesthésies avec $10 \mathrm{mg}$ de bupivacaïne sans complication apparente. Le deuxième patient souffrant d'une légère diminution de la force motrice des deux membres inférieurs suite à une poliomyélite, subi une perte de fonction motrice permanente ad $\mathrm{L}_{3} \mathrm{du}$ côté gauche et $\mathrm{L}_{1}$ du côté droit après une péridurale pour réduction ouverte d'une fracture de la diaphyse fémorale.

Finalement, avant d'étendre les blocs nerveux continus aux patients porteurs de pathologie neurologique ou musculaire il faudra en connaitre davantage sur la myotoxicité des anesthésiques locaux. ${ }^{17}$ Les anesthésiques locaux peuvent induire des myonécroses. Mis à part l'atteinte des muscles extraoculaires (blocs péri- et rétrobulbaires) il en résulte toutefois rarement un problème clinique décelable. Étant donné que les myoblastes et les éléments du tissu conjonctif demeurent intacts, ce phénomène est habituellement quasi totalement réversible et une régénération survient entre trois et quatre semaines. L'effet des perfusions d'anesthésiques locaux sur un muscle anormal n'est toutefois pas connu.

En conclusion il est possible que les patients porteurs de pathologie neurologique incluant ceux avec polyneuropathie diabétique sévère soient plus susceptibles aux complications neurologiques de l'anesthésie régionale. Le risque pourrait varier selon le type de pathologie et la sévérité de l'atteinte. Il n'est pas actuellement possible de quantifier l'ampleur de l'accroissement de l'incidence. Pour obtenir une puissance adéquate, la réalisation d'essais randomisés à cet effet nécessiterait probablement l'inclusion de plusieurs milliers de patients et la randomisation des patients à l'une ou l'autre des techniques poserait à n'en pas douter des problèmes éthiques complexes. En dépit de leurs limites méthodologiques, les études rétrospectives demeurent actuellement la meilleure évidence disponible. Les études de cas ou les séries de cas comme celle publiée dans ce numéro du 
Journal apportent des renseignements utiles sur la faisabilité de la technique chez ces patients à partir de l'expérience d'un centre. ${ }^{9}$ En examinant la meilleure évidence disponible permettant de choisir la technique anesthésique chez un patient porteur de pathologie neurologique, il faut aussi tenir compte des risques de complications inhérents à l'anesthésie générale. Des patients porteurs de la maladie de Charcot-MarieTooth ont été opérés avec succès sous anesthésie régionale (blocs nerveux périphériques ou anesthésie périmédullaire) ou générale. ${ }^{9,18}$ Une patiente a toutefois due être ventilée mécaniquement pendant près d'un mois après une simple césarienne sous anesthésie générale. ${ }^{19}$ Finalement, il faut aussi tenir compte du degré d'expertise de l'opérateur avant de déterminer si la guidance par ultrasons des techniques d'anesthésie régionale apportera ou non un bénéfice chez les patients porteurs de pathologie neurologique.

\section{References}

1 Brull R, McCartney CJ, Chan VW, El-Beheiry H. Neurological complications after regional anesthesia: contemporary estimates of risk. Anesth Analg 2007; 104: 965-74.

2 Lee LA, Posner KL, Domino KB, Caplan RA, Cheney $F W$. Injuries associated with regional anesthesia in the 1980s and 1990s: a closed claims analysis. Anesthesiology 2004; 101: 143-52.

3 Horlocker TT, Kufner RP, Bishop AT, Maxson PM, Schroeder DR. The risk of persistent paresthesia is not increased with repeated axillary block. Anesth Analg 1999; 88: 382-7.

4 Deschner S, Borgeat A, Hadzic A. Neurologic complications of peripheral nerve blocks: mechanisms and management. In: Hadzic A (Ed.). Textbook of Regional Anesthesia and Acute Pain Management. New York: McGraw-Hill; 2007: 969-96.

5 Upton AR, McComas AJ. The double crush in nerve entrapment syndromes. Lancet 1973; 2: 359-62.

6 Hebl JR, Horlocker TT, Sorenson EJ, Schroeder DR. Regional anesthesia does not increase the risk of postoperative neuropathy in patients undergoing ulnar nerve transposition. Anesth Analg 2001; 93: 1606-11.

7 Delaunay L, Chelly JE. Blocks at the wrist provide effective anesthesia for carpal tunnel release. Can J Anesth 2001; 48: 656-60.

8 Hebl JR, Horlocker TT, Pritchard DJ. Diffuse brachial plexopathy after interscalene blockade in a patient receiving cisplatin chemotherapy: the pharmacologic double crush syndrome. Anesth Analg 2001; 92: 249-51.

9 Dhir S, Balasubramanian S, Ross D. Ultrasound guided peripheral regional blockade in patients with Charcot-
Marie-Tooth disease: a review of three cases. Can J Anesth 2008: 55: 515-20.

10 Koff MD, Cohen JA, McIntyre JJ, Carr CF, Sites BD. Severe brachial plexopathy after an ultrasoundguided single-injection nerve block for total shoulder arthroplasty in a patient with multiple sclerosis.

Anesthesiology 2008; 108: 325-8.

11 Sites BD, Spence BC, Gallagher JD, Wiley CW, Bertrand $M L$, Blike GT. Characterizing novice behavior associated with learning ultrasound-guided peripheral regional anesthesia. Reg Anesth Pain Med 2007; 32: 107-15.

12 Perlas A, Chan VW, Simons M. Brachial plexus examination and localization using ultrasound and electrical stimulation: a volunteer study. Anesthesiology 2003; 99: 429-35.

13 Bigeleisen PE. Nerve puncture and apparent intraneural injection during ultrasound-guided axillary block does not invariably result in neurologic injury. Anesthesiology 2006; 105: 779-83.

14 Hebl JR, Horlocker TT, Schroeder DR. Neuraxial anesthesia and analgesia in patients with preexisting central nervous system disorders. Anesth Analg 2006; 103: 223-8.

15 Hebl JR, Kopp SL, Schroeder DR, Horlocker TT. Neurologic complications after neuraxial anesthesia or analgesia in patients with preexisting peripheral sensorimotor neuropathy or diabetic polyneuropathy. Anesth Analg 2006; 103: 1294-9.

16 Aldrete JA, Reza-Medina M, Dand O, et al. Exacerbation of preexisting neurological deficits by neuraxial anesthesia: report of 7 cases. J Clin Anesth 2005; 17: 304-13.

17 Zink W, Graf BM. Local anesthetic myotoxicity. Reg Anesth Pain Med 2004; 29: 333-40.

18 Greenwood JJ, Scott WE. Charcot-Marie-Tooth disease: peripartum management of two contrasting clinical cases. Int J Obstet Anesth 2007; 16: 149-54.

19 Brian JE Jr, Boyles GD, Quirk JG Jr, Clark RB. Anesthetic management for cesarean section of a patient with Charcot-Marie-Tooth disease. Anesthesiology 1987; 66: 410-2. 